\title{
Peer Reviewing Interdisciplinary
}

\section{Papers}

\author{
MARCO PAUTASSO* and CESARE PAUTASSO** \\ *Division of Biology, Imperial College London, Silwood Campus, Ascot SL5 \\ 7PY, UK. E-mail: m.pautasso@ic.ac.uk; ${ }^{* *}$ Faculty of Informatics, University \\ of Lugano, Via Buffi 13, 6900 Lugano, Switzerland
}

Interdisciplinary research is becoming more frequent because many contemporary issues can only be successfully addressed by integrating different perspectives. One general feature of the various scientific fields is peer review, i.e. the assessment and improvement of submissions to journals, conferences and workshops. Whilst there exist guidelines for the peer review of monodisciplinary articles and empirical studies of how interdisciplinary research proposals are assessed, there is still a need for a summary of issues specific to the peer review of interdisciplinary research papers. This article provides an overview of relevant questions such as whether reviewers are competent to assess interdisciplinary papers even if unfamiliar with all the involved fields. We discuss the assessment of the interdisciplinarity, soundness, novelty, influence and general interest of interdisciplinary manuscripts. Further issues include the appropriateness of interdisciplinary submissions for journals, keeping the vocabulary of new interdisciplinary fields understandable to the reader and balancing the references across various fields. Constructive interdisciplinary reviewers are likely to be just as open-minded as interdisciplinary scientists and should be rewarded more than they currently are.

\section{Introduction}

Although slowly, science is indeed becoming more interdisciplinary (Figure 1). ${ }^{1,2}$ Many contemporary issues need to be tackled from multiple points of view to be thoroughly investigated and successfully solved/addressed. Examples include climate change, environmental pollution, happiness, mobility, poverty, public health and sustainability. ${ }^{3,4}$ Interdisciplinary research integrates concepts, tools and/or information from two or more established fields. These fields can be neighbouring 


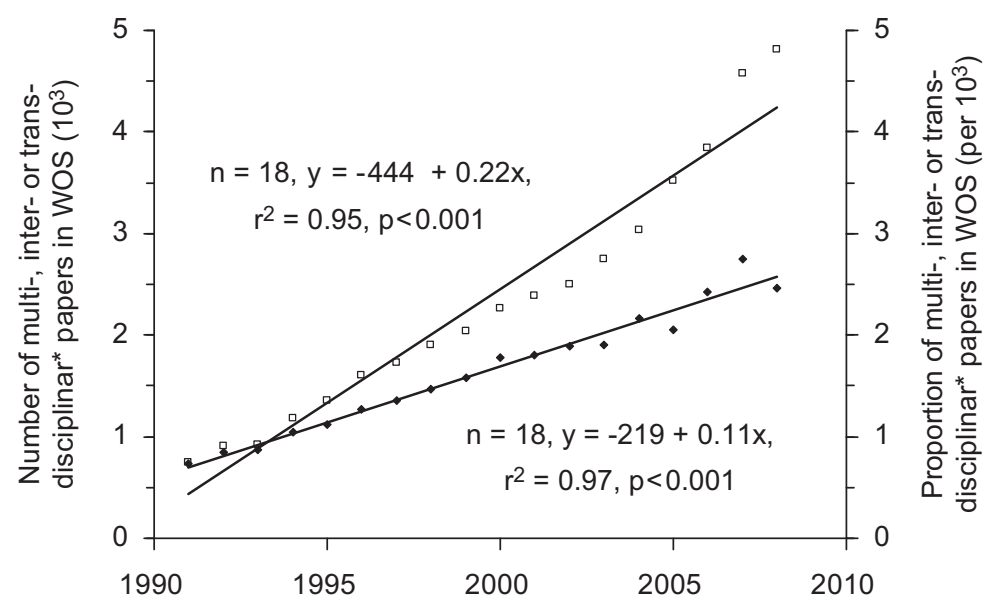

Figure 1. The absolute (empty squares) and relative (filled diamonds) increase of the multi-, inter- or transdisciplinary peer-reviewed literature (1991-2008, the period for which abstracts are searched; data from ISI (C), Web of Science, all Citation Indexes, as of April 2009)

disciplines such as plant and forest pathology, or can be located far apart in the scientific landscape (e.g. informatics and psychology, physics and dendrology, climate science and anthropology).

One of the practices common to the various scientific fields is peer review. Peer review is a process that aims (i) to identify submissions worthy of publication (particularly in a time of increasing rejection rates due to the 'publish or perish' system), ${ }^{5}$ and (ii) to help improve drafts in their content and form. ${ }^{6,7}$ At its best, peer review can act as a powerful filter that avoids busy readers wasting their time reading untrustworthy, unintelligible and/or unoriginal research. Since peer review makes it less easy to publish reinventions of the steering wheel and complicated studies of frivolous issues, it can also contribute to keeping eggs off authors' faces. ${ }^{8,9}$

Guidelines on how to peer review mono-disciplinary research papers have appeared in various fields, from computer science ${ }^{10}$ to medicine. ${ }^{11}$ Such articles also provide useful suggestions for peer reviewers of interdisciplinary papers, but are not specifically written with that audience in mind. There have been some empirical analyses of how interdisciplinary research proposals are judged by selection panels. ${ }^{12,13}$ These studies can be helpful to discover the problems involved with the assessment of proposals that go beyond the traditional boundaries of university departments. However, they are not thought through from the perspective of the reviewers of interdisciplinary research that has already been accomplished (rather than proposed for the future). A short note on the issue of interdisciplinary peer review has appeared in the Nature online debate on peer review, ${ }^{14}$ but there is still a need for an article focusing on the 
specific issues of the peer review of interdisciplinary papers, from the skills needed to assess interdisciplinary submissions, to issues such as the soundness, novelty and interest of an interdisciplinary paper.

\section{Is the reviewer able to assess an interdisciplinary paper?}

Before agreeing to assess a manuscript, reviewers should ask themselves whether they feel competent enough to do so. ${ }^{7,15}$ This is likely to be a thorny issue for interdisciplinary submissions, particularly for innovative approaches. In this case, few scientists will be equally familiar with all of the fields spanned by the article. Thus, many reviewers will only be able to provide a partial assessment from the point of view of their own discipline or methodological approach. ${ }^{8,14,16}$ In this situation, the reviewer can still provide a useful report, but should make it clear in the confidential comments to the editorial team that she has commented on, for example, the mathematical methodology, and left, for example, the biological framework to be checked by someone else.

There are further reasons to accept to review an interdisciplinary paper even if the reviewer is not an active researcher in all the relevant fields. One reason is to get to know about new interdisciplinary developments in advance and with much more depth than just by quickly scanning the abstract of the published paper. Secondly, reviewing an interdisciplinary paper is an exercise in thinking out of the box and is thus good practice in sharpening one's own creative thinking. Moreover, the editor might be looking for an opinion from outside the interdisciplinary field and thus for a more detached view on the analysis. Although being few in number, specialists of, say, the economics of space tourism, will tend to be enthusiastic about the topic and eager to promote it. Paraphrasing Smith, ${ }^{10}$ this is why an editor who wants to debunk a paper on constructivist alchemy sends it to a mathematical chemist, not to an alchemist, a constructivist or a combination of the two.

In general, assessing interdisciplinary papers requires personal characteristics that also favour the pursuit of interdisciplinary research (e.g. open-mindedness, flexibility, curiosity, lateral thinking). ${ }^{1}$ Even if the reviewer is not an active researcher in the particular interdisciplinary field of the submission, it can help if she has already worked in an interdisciplinary way, even if in other fields. Although this remains to be investigated and exceptions will be common, it could be that, other things being equal, speaking more than one language and/or having lived in different countries, by providing more opportunities for intercultural experiences, could be associated with better skills in assessing interdisciplinary research. ${ }^{17}$

\section{Is the paper really interdisciplinary?}

If the submission claims to be interdisciplinary (either explicitly in title, abstract and/or text, or implicitly, by having been submitted to a journal publishing 
interdisciplinary papers), then it is important that the reviewer makes sure that this is indeed the case. Since interdisciplinarity is generally seen as a good thing, ${ }^{18}$ it could be surreptitiously used by authors to increase their chances of acceptance.

A reviewer should thus ask: is this paper really making use of the theories and/or methods of several fields? Is it integrating them or merely juxtaposing them? In the latter case, the analysis would probably be better described as multirather than inter-disciplinary, although use of these terms is probably not consistent outside of the literature on multi- and inter-disciplinarity. ${ }^{19,20}$ Therefore, reviewers should check that the papers correctly identify their contribution as being inter-, multi-, or even trans-disciplinary. Should the article transcend the fields by creating a hitherto unused approach, it might be not just inter-, but transdisciplinary. ${ }^{1}$ This is not the case if the authors only use the methods of one field to address a problem normally tackled in another discipline. Sometimes interdisciplinary approaches become so common that they create a new field (e.g. bio-informatics, molecular ecology, network epidemiology). One of the signs that these have become established boundary fields is when articles on these topics stop claiming explicitly to be interdisciplinary and journals specialized in the new interdisciplinary fields appear.

\section{Is the interdisciplinary paper sound?}

Whether or not there is an explicit claim of interdisciplinarity, the judgement of whether an interdisciplinary manuscript should be published or not has to rest on other features. A poorly executed analysis needs revision even if it is a classic example of interdisciplinary research. This may, however, turn out not to be trivial to detect: in newly forming interdisciplinary fields, the boundaries between rigorous and poor science may become fuzzy. After all, this is the point of interdisciplinary research: questioning established practices and introducing new ways of looking at scientific problems.

How to assess scientific integrity in these cases? Questions such as whether the statistics follow the best current practices, the results appear to be plausible, and the authors did what they claim to have done ${ }^{10}$ remain relevant for interdisciplinary papers. Different scientific fields may have different standards as to what makes a good contribution to the literature, ${ }^{16,21}$ but there are general features (e.g. a long-awaited solution to an unsolved problem, comprehensive empirical evidence/theoretical framework, balanced discussion of previous relevant literature) which are shared among most fields. To gauge the scientific soundness, a reviewer may also make use of ancillary features of the article, such as redundancy in the text, quality of the figures, presence/absence of motivation and evaluation for the study. However, can the quality of the overall reasoning of a manuscript be used to infer the soundness of an interdisciplinary approach? 
Logical leaps, muddled sentences, inconsistencies are all unfortunate features of a submission, but may not be compelling evidence against performing and reporting an innovative analysis, as they can all be avoided in a revised manuscript.

On the other hand, given that journals are nowadays flooded with submissions, ${ }^{7,22}$ features such as poor use of statistical tests, failure to motivate the choice of a particular technique and absence of a framework of related studies justifying the question investigated can be fatal even to the most original interdisciplinary study. If an article is clearly interdisciplinary and sound, so that it deserves publication after problems in the presentation have been solved, then reviewers can be very helpful by pointing out, for example, discrepancies between abstract and text, lack of clarity of particular sentences, alternative explanations for the results or unwarranted conclusions. ${ }^{9}$ In the following, we discuss in the context of interdisciplinary contributions some key criteria generally used in the assessment of mono-disciplinary submissions.

\section{Is the interdisciplinary paper original?}

Novel hypotheses, methodology, results and/or conclusions are important features of submissions, and may make such work likely to be influential, if coupled with addressing a non-trivial issue. Interdisciplinary papers are likely to be original, but are not necessarily so. There can be interdisciplinary papers that repeat or confirm interdisciplinary analyses already present in the literature, whether the authors knew about these previous studies or not. A reviewer of an interdisciplinary submission is thus well advised to check the literature for analogous studies. In some fields, making sure that similar analyses have not already been done can entail a literature search spanning decades. For novel interdisciplinary approaches, reviewers have to be particularly up-to-date with recent papers, which can mean having to scan the online lists of papers in press of relevant journals, since these papers will tend not yet to be present in databases. It can also be helpful looking at previous papers from the same group of authors. ${ }^{8}$

Although one can never be sure that something has not already been done, there are now tools that enable one to search the scientific literature in ways which were undreamt of only a few decades ago (Web of Science, ISI Proceedings, Medline, Scopus, Google Scholar, DBLP). ${ }^{23-28}$ A diligent reviewer will thus make use of more than one database. It might be easier to scan the literature in certain interdisciplinary fields if these fields have just been launched. However, in some cases, similar approaches may exist that are not called the same thing, so that reviewers of interdisciplinary manuscripts need to be creative with the keywords they use. Despite the growing proportion of scientific studies published in English, ${ }^{29}$ in certain cases it might still be worthwhile searching in the scientific literature of other languages. 


\section{Is the interdisciplinary paper likely to inspire further research?}

It might be years before it becomes clear whether a paper was influential or not, but journals are still interested in publishing papers that are likely to inspire further research. This is because of the tyranny of the impact factor, which provides a positive feedback between influential articles in a given journal and more influential articles in later volumes of the same journal. Original interdisciplinary papers are likely to be influential, as they open up new perspectives and ways of doing science.

This can make journals wishing to increase their standing eager to publish interdisciplinary submissions. At the same time, given the high rejection rates of many journals and the pressure to publish, authors may try to exploit this situation by exaggerating their claims of interdisciplinarity. A reviewer thus needs to double-check carefully that a paper which claims to be interdisciplinary really is so, not just for the sake of assessing the correctness of such a claim, but also because interdisciplinarity is likely to inspire further research. Of course, this may not be sufficient, as influential papers also need to be sound, original and address a problem on which many people are likely to go on working.

Just as for non-interdisciplinary submissions, the likelihood that an interdisciplinary paper will be influential depends on whether it presents major results or not. Elegant and technically correct but useless interdisciplinary work; positive, but minor, multidisciplinary contributions to knowledge; and neither elegant nor useful, but not actually wrong transdisciplinary analyses are all typically less likely to produce an outstanding and long-remembered publication with enthusiastic support from reviewers. ${ }^{10}$ A problem with assessing interdisciplinary submissions, having at the back of the mind the likelihood that they will be influential, can be that this might produce bias in the literature. It is known that submissions that report the presence of significant differences are more likely to be published and to be cited than those that fail to do so. ${ }^{30,31}$ If reviewers judge more favourably exciting (as opposed to unsurprising) interdisciplinary papers in the same way as reviewers of non-interdisciplinary papers appear to do, ${ }^{32}$ the file drawer problem (positive results are more likely to be published than negative ones) will also be replicated for the interdisciplinary literature.

\section{Is the interdisciplinary paper of broad interest?}

A novel and influential paper is in many cases a paper of broad interest. This is because it will be read (and cited) widely. However, a paper may be influential in a certain community without necessarily becoming known in other fields or by the public at large. Interdisciplinary papers have the advantage of spanning more than one discipline, thus multiplying the potential number of readers. Still, interdisciplinarity does not necessarily guarantee broad interest. A paper in, for example, ethnobotany may be specialized for readers of that interdisciplinary field and of little appeal even to pure ethnologists or botanists. 
For an interdisciplinary paper to be of broad interest it needs to be required reading to researchers in the two or more parent disciplines, and to be of general interest to other scientists. Today, this is a tough requirement, given the magnitude of the scientific literature produced yearly in any field of inquiry. Many journals of high reputation now routinely (and not very imaginatively) motivate their rejections with the too narrow interest of the submission. Interdisciplinarity can help in broadening the audience of a paper, but may still not be enough to justify acceptance in such journals. As interdisciplinarity becomes more common, it inevitably loses some of its sparkle. Reviewers for journals with high rejection rates thus need to watch out whether interdisciplinarity is accompanied by other features such as novelty, appropriateness and generality of the approach. They should also try to predict whether the paper would appeal to a readership found across the union of the multiple fields as opposed to the intersection.

\section{Is the paper appropriate for the journal?}

There is a feeling among scholars of interdisciplinarity that studies at the boundary of scientific fields have more trouble in finding an appropriate journal than do mono-disciplinary submissions. ${ }^{33}$ This is not so much the case for established interdisciplinary fields such as astrobiology, bioinformatics, conservation biogeography or clinical biochemistry, but can be so for those recently formed or merging perspectives from distantly related fields (e.g. remote sensing and linguistic anthropology). In the latter case, scientists may find it hard to publish the results of their interdisciplinary collaborations because a journal for their new field does not yet exist and the journals of the two or more parent disciplines do not recognize such articles as appropriate.

Reviewers of interdisciplinary submissions should try to balance the requirements of specialized journals with the advantages provided by new perspectives and cooperation with other fields. If an interdisciplinary contribution is original, wellresearched and likely to lead to publicity in the press, publication should be recommended even if the journal to which it was submitted does not explicitly welcome studies merging perspectives with neighbouring or distant fields. At the same time, a submission that claims to be at the interface between, for example, the life and the physical sciences is only appropriate for a journal that publishes such submissions if it contains both perspectives. Reviewers should ensure that such a contribution is well balanced, with no predominance of one of the two perspectives.

\section{Is the paper understandable?}

Interdisciplinary articles are at risk of not being understood by practitioners of the two (or more) parent fields, as they integrate new methods and concepts. After all, one of the reasons most scientists tend to remain active in a certain field rather 
than jumping from, for example, software engineering to renewable energy policy or from plant genetic resources to internal medicine is that there is a cost inherent in such career moves. This involves getting to learn new bodies of knowledge with their associated underpinning theories, outstanding issues and shared vocabularies. Interdisciplinary science is a way of building bridges between scientific fields and can thus enhance communication where this was absent or rare. However, hybridization between scientific fields can also create a new jargon inaccessible to the uninitiated.

Reviewers should make sure they understand what is written in an interdisciplinary manuscript. If they cannot follow its vocabulary, chances are high that readers will also not make much out of the published article. Although it is not the task of a reviewer to rewrite a draft for the authors, suggestions as to which parts can be better formulated and what general issues make understanding of a particular interdisciplinary submission difficult are all features of constructive reviews. ${ }^{8,10}$ A reviewer coming from one of the two (or more) disciplines involved is obviously in a better position to assess and, if needed, improve the parts of the manuscript relevant to his or her discipline(s). However, that reviewer should still read the remaining parts of the text so as to ensure that at least the gist of it can be understood by a reader not entirely familiar with the subject.

\section{Are the references interdisciplinary?}

A balance between the two (or more) parent disciplines also needs to be achieved in terms of the cited references. These should not just reflect one or the other field. Similarly, the references cited in a paper on, for example, emergency medicine and developmental economics should span a similar temporal period for both fields, without being all recent for one and all from the 1970s for the other. In established interdisciplinary fields at the interface between, for example, the mathematical and the biological sciences, this issue may not present itself, as there will be no need to go back to the single disciplines given the availability of previous studies in the interdisciplinary field.

In general, just as for non-interdisciplinary submissions, a reviewer of interdisciplinary articles should comment on whether the authors have made reference to appropriate work, and whether there are too many (or not enough) references. However, interdisciplinary reviewers who wish to maintain their anonymity (e.g. Smith) should take care to avoid suggesting that the authors cite more papers by Smith et al. This can be a problem in the case of interdisciplinary manuscripts if the field is still very young and there have only been a few previous studies using a particular approach. In this case, the recommendation that the paper by Smith should be referred to might not actually indicate that the reviewer of the submitted paper was Smith. 


\section{Conclusions}

Interdisciplinarity is becoming more important in science. Assessing interdisciplinary contributions to the literature can provide a challenge to reviewers, even more so when reviewers are already struggling to find the time to read mono-disciplinary manuscripts. ${ }^{22,34}$ Many of the general issues to which peer reviewers of mono-disciplinary submissions should pay attention (e.g. novelty, flow, accuracy, precision, correctness, consistency, length, clarity) are also relevant in the case of interdisciplinary papers. There are some issues specific to the peer review of interdisciplinary articles - for example (1) whether the paper is really interdisciplinary, (2) the balance between fields, (3) the use of new vocabulary - but these need not discourage scientists from the task, even if they are not familiar with both of the parent disciplines of the interdisciplinary field. Peer reviewing interdisciplinary papers requires open-mindedness, but can also foster it and is thus probably more intellectually rewarding than assessing mono-disciplinary manuscripts. However, if peer review has to retain its value in the face of the explosion in scientific publications, the development of the internet and the open access movement, ${ }^{35}$ the drive towards quantitative research evaluation ${ }^{36}$ and other structural changes in the production of knowledge (e.g. interdisciplinarity), devoting time and energy to the quality assessment of submissions to journals should be better rewarded than has traditionally been the case.

\section{Acknowledgements}

Many thanks to L. Ambrosino, R. Brown, T. Hirsch, O. Holdenrieder, M. Jeger, D. Liggins, C. Lyall and the participants of the workshop on 'How to Peer Review Research Papers' at Imperial College for insights and discussion, and to T. Matoni for helpful comments on a previous draft.

\section{References}

1. A. Bruce, C. Lyall, J. Tait and R. Williams (2004) Interdisciplinary integration in Europe: the case of the Fifth Framework programme. Futures, 36, 457-470. doi:10.1016/j.futures.2003.10.003.

2. A. L. Porter and I. Rafols (2009) Is science becoming more interdisciplinary? Measuring and mapping six research fields over time. Scientometrics, 81, 719-745. doi:10.1007/s11192-008-2197-2.

3. A. Buanes and S. Jentoft (2009) Building bridges: institutional perspectives on interdisciplinarity. Futures, 41, 446-454. doi:10.1016/j.futures.2009.01.010.

4. C. Pohl (2005) Transdisciplinary collaboration in environmental research. Futures, 37, 1159-1178. doi:10.1016/j.futures.2005.02.009.

5. P. A. Lawrence (2003) The politics of publication - authors, reviewers and editors must act to protect the quality of research. Nature, 422, 259-261. doi:10.1038/422259a.

6. B. K. Forscher (1964) Rules for referees. Science, 150, 319-321. 
7. S. Goldbeck-Wood (1998) What makes a good reviewer of manuscripts? British Medical Journal, 316, 86.

8. F. G. Hoppin (2002) How I review an original scientific article. American Journal of Respiratory and Critical Care Medicine, 166, 1019-1023. doi:10.1164/rccm.200204-324OE.

9. P. E. Bourne and A. Korngreen (2006) Ten simple rules for reviewers. PLoS Computational Biology, 2, e110. doi:10.1371/journal.pcbi.0020110.

10. A. J. Smith (1990) The task of the referee. IEEE Computer, 23, 46-51.

11. J. M. Provenzale and R. J. Stanley (2005) A systematic guide to reviewing a manuscript. American Journal of Radiology, 185, 848-854. doi:10.2214/ AJR.05.0782.

12. G. Laudel (2006) Conclave in the Tower of Babel: how peers review interdisciplinary research proposals. Research Evaluation, 15, 57-68.

13. V. B. Mansilla (2006) Assessing expert interdisciplinary work at the frontier: an empirical exploration. Research Evaluation, 15, 17-29.

14. C. Lee (2006) Perspective: peer review of interdisciplinary scientific papers. Nature, online debate on peer review, doi:10.1038/nature05034.

15. P. Rosenbaum (2005) On the value of being a journal reviewer. Developmental Medicine \& Child Neurology, 47, 147doi:10.1017/ S0012162205000265.

16. E. De Schutter (2008) Reviewing multi-disciplinary papers: a challenge in neuroscience? Neuroinformatics, 6, 253-255. doi:10.1007/s12021-008-9034-x.

17. K. Lasater, A. L. Ray, M. J. Driever, A. Rosenfeld and K. J. Bradley (2008) Creating international conference submission and review guidelines to facilitate transnational dialogue. The Journal of Continuing Education in Nursing, 39, 473-479.

18. J. T. Klein (2006) Afterword: the emergent literature on interdisciplinary and transdisciplinary research evaluation. Research Evaluation, 15, 75-80.

19. T. Ramadier (2004) Transdisciplinarity and its challenges: the case of urban studies. Futures, 36, 423-439. doi:10.1016/j.futures.2003.10.009.

20. D. A. Booth (2008) Appetite: peer-reviewed research across the disciplines. Appetite, 51, 231-232. doi:10.1016/j.appet.2008.04.005.

21. J. T. Klein (2008) Evaluation of interdisciplinary and transdisciplinary research. A literature review. American Journal of Preventive Medicine, 35, S116-S123. doi:10.1016/j.amepre.2008.05.010.

22. M. E. Hochberg, J. M. Chase, N. J. Gotelli, A. Hastings and S. Naeem (2009) The tragedy of the reviewer commons. Ecology Letters, 12, 2-4. doi:10.1111/j.1461-0248.2008.01276.x.

23. H. M. Müller, E. E. Kenny and P. W. Sternberg (2004) Textpresso: an ontology-based information retrieval and extraction system for biological literature. PLoS Biology, 2, 1984-1988. doi:10.1371/journal.pbio.0020309.

24. H. M. Müller, R. Rangarajan, T. K. Teal and P. W. Sternberg (2008) Textpresso for neuroscience: searching the full text of thousands of neuroscience research papers. Neuroinformatics, 6, 195-204. doi:10.1007/ s12021-008-9031-0. 
25. J. L. Fink, S. Kushch, P. R. Williams and P. E. Bourne (2008) BioLit: integrating biological literature with databases. Nucleic Acids Research, 36, W385-W389. doi:10.1093/nar/gkn317.

26. M. Errami, Z. H. Sun, T. C. Long, A. C. George and H. R. Garner (2009) Deja vu: a database of highly similar citations in the scientific literature. Nucleic Acids Research, 37, 921-924. doi:10.1093/nar/gkn546.

27. D. W. Lawrence and L. Laflamme (2009) Using online databases to find journal articles on injury prevention and safety promotion topics: how do SafetyLit subscribers use other databases? Safety Science, 47, 1-8. doi:10.1016/j.ssci.2008.01.004.

28. F. Lufrano and P. Staiti (2009) A bibliometric analysis of the international literature in supercapacitors. International Journal of Electrochemical Science, 4, 173-186.

29. P. E. Valkimadi, D. E. Karageorgopoulos, H. Vliagoftis and M. E. Falagas (2009) Increasing dominance of English in publications archived by PubMed. Scientometrics, 81, 219-223. doi:10.1007/s11192-008-2139-z.

30. C. B. Begg and J. A. Berlin (1988) Publication bias: a problem in interpreting medical data. Journal of the Royal Statistical Society Series A, 151, 419-463.

31. P. J. Easterbrook, J. A. Berlin, R. Gopalan and D. R. Matthews (1991) Publication bias in clinical research. Lancet, 337, 867-872.

32. M. Jasienski (2009) Garfield's demon and 'surprising' or 'unexpected' results in science. Scientometrics, 78, 347-353. doi:10.1007/s11192-0071979-2.

33. G. L. A. Fry (2001) Multifunctional landscapes - towards transdisciplinary research. Landscape and Urban Planning, 57, 159-168. doi:10.1016/ S0169-2046(01)00201-8.

34. M. Hauser and E. Fehr (2007) An incentive solution to the peer review problem. PLoS Biology, 5, e107. doi:10.1371/journal.pbio.0050107.

35. D. Imboden (2009) Scientific publishing: the dilemma of research funding organizations. European Review, 17, 23-31. doi:10.1017/ S1062798709000544.

36. D. Weaire (2007) Time for a rethink of research proposal evaluation? European Review, 15, 275-282. doi:10.1017/S1062798707000300.

\section{About the Authors}

Marco Pautasso is a postdoc in network epidemiology and is also interested in conservation biogeography and landscape pathology.

Cesare Pautasso is an assistant professor in informatics, with research interests in, for example, software composition for distributed systems, service-oriented architecture and web services. 